\title{
Arte e investigación
}

$\mathrm{D}$ ebido a las características de esta investigación es necesario acudir a una metodología que no se base en las exactitudes, en datos numéricos, en mediciones o control de variables, sino que por el contrario colabore en la recolección de información a través de narrativas, imágenes, texturas, historias y así mismo permita su análisis; es por ello que la metodología utilizada es cualitativa.

Según Taylor y Bogdan (1984), la metodología cualitativa parte de desarrollar conceptos y comprensión a partir de la interpretación de pautas en los datos y no parte de estos últimos para evaluar modelos preconcebidos, hipótesis o teorías. Siendo así, los estudios cualitativos tienen como base ideas e interrogantes vagamente planteados. En el caso de esta investigación, no hay un planteamiento de hipótesis o un interés en comprobar o afirmar una teoría, el objetivo apunta a promover procesos de autoconocimiento y autorregulación a través de un programa curricular basado en la realización de talleres creativos que tienen como soportes actividades artísticas con enfoque terapéutico, por lo tanto se requiere de un análisis netamente cualitativo de la información; y acudiendo al método inductivo, en el que se parte de lo particular a lo general, para que los resultados obtenidos permite realizar conclusiones generalizables al contexto espacial e histórico en el que se realizó la investigación. 
Con esta última idea es importante aclarar que, si bien se pueden generalizar los resultados de la investigación para modificaciones curriculares en la educación de los jóvenes, no se pretende generar verdades universales o conclusiones acabadas, sino que este estudio es parte de un proceso que puede continuarse y está en constante transformación, cambio y construcción de nuevo conocimiento.

En esta investigación hay un interés por analizar de manera total la información y no segmentarla, sino por dar cuenta de un fenómeno en su totalidad: las actividades artísticas y el aporte de estas a los jóvenes en su dimensión como humanos. Al respecto Taylor y Bogdan (1984) mencionan que las personas, escenarios o grupos no son reducidos a variables, sino que se consideran un todo que es interpretado en su contexto; el investigador cualitativo considera todas las perspectivas valiosas, ya que en la población que estudia, no hay voces más importantes que otras y no anda en búsqueda de la verdad o de la moralidad, sino de una comprensión detallada de las perspectivas de otras personas.

Taylor y Bogdan (1984) dicen que las investigaciones cualitativas son humanistas, ya que influyen en el modo como las vemos; al acercarnos a la realidad de un ser humano, hay un nivel de comprensión de su sentir y experiencias que no es posible conocer cuando se trabaja con datos estadísticos. Martínez (2011) menciona que en la investigación cualitativa cobra importancia la relación investigador-sujetos y es justo esa dinámica - la que genera los problemas de la ciencia- lo que aplica a la presente investigación, la cual tiene un carácter netamente humano en el sentido de facilitar el espacio de introspección para los estudiantes y también considera a los participantes como humanos en una relación entre ellos y con el investigador, quien tiene una visión particular sobre su objeto de investigación.

Por sus implicaciones, Taylor y Bogdan (1984) afirman que la investigación cualitativa es un arte, es flexible en cuanto al modo de realizar los estudios y el investigador es alentado a crear su propio método. En relación con esta idea, Albertín (2005) afirma que la metodología cualitativa intenta comprender el sentido y los significados que tienen los procesos y fenómenos sicológicos y sociales para los actores implicados. Los resultados están muy vinculados al contexto en el que se 
producen las acciones, pero se relacionan con contextos de orden más social e histórico como marco de interpretación. Es así que, al pensar que en esta investigación era necesario realizar comprensiones sobre los jóvenes y la arteterapia teniendo como marco el tiempo actual y las situaciones que les acontecen a los participantes, hay una necesidad de acudir a la metodología cualitativa.

\section{La investigación-acción como método de investigación}

Partiendo de una metodología cualitativa para la realización de esta investigación, el método que permitía acercarse a los estudiantes y conocer en cierta manera sus procesos con la arteterapia, la posibilidad de autorregularse y autoconocerse es la investigación-acción (I-A). Por ello es importante revisar algunas generalidades de este método y explicar cómo se conecta con los objetivos de la investigación.

Según Rojas (2012), la I-A es un método propuesto por Kurt Lewin en la década de los años cuarenta, que se enmarca en las ciencias sociales y propone favorecer el diálogo y la participación entre los investigados y el investigador; busca generar caminos para comprender una realidad concreta y propone intervenciones que mejoren la calidad de vida de la población con la que se está trabajando. En este tipo de investigación el rol del investigador consiste en acercarse a la comunidad o grupo por investigar e involucrarse con ellos, fomentando la participación de los mismos en la búsqueda de información y el análisis de los resultados, es por esto que el conocimiento y la comprensión que se hayan realizado apuntan a la transformación social de la comunidad investigada.

Al pensar que la presente investigación tuvo como población diferentes grupos de estudiantes de educación superior y que la investigadora se acercó a ellos desde una postura docente, se puede pensar que la investigadora se involucró en el grupo y no tenía tan solo el rol de observador; la investigadora estaba en contacto con los estudiantes y participaba de las clases interactuando con ellos; además, los propios 
participantes alimentaban la investigación con sus intervenciones, los productos que realizaban y el resultado del estudio apunta a transformar la realidad del currículo universitario en la carrera y universidad en donde se investigó, y por lo tanto busca mejorar la calidad de vida de los estudiantes.

Rojas (2012) menciona que el objetivo de la I-A es proponer acciones, transformar la realidad de un grupo en búsqueda de su beneficio, por lo que la teoría está fuertemente ligada a la práctica, las ideas se vinculan a las acciones y estas se revisan para determinar si son las adecuadas o deben cambiarse.

\section{Características de la investigación-acción}

De manera resumida, la I-A tiene las siguientes características:

- Es una experiencia concreta, real, de un grupo de sujetos; no es una formulación a priori o estrictamente teórica.

- La problemática se construye a partir de procesos deliberativos y se asienta en un diagnóstico participativo que se construye con los propios implicados.

- Los investigadores trabajan con sujetos en contextos reales.

- Busca un cambio concreto en los sujetos con los que trabaja e investiga.

- En la formulación de los objetivos, la comunidad investigada participa, opina y se vincula activamente en la propuesta de investigación.

- Los investigadores no solo observan, además participan y se comprometen con la realidad que están investigando.

- Es una investigación de escala reducida, local.

- Levanta información que puede ser sistematizada y servir para explicar fenómenos más generales.

- Propone líneas de acción para intervenir en la realidad analizada. 
- Se somete permanentemente a los juicios de los implicados (investigadores/sujetos investigados) (Oliveira, citado en Rojas, 2012, p. 4).

Kemmis y MacTaggart (1998, citados en Baucela, 2004) agregan que la I-A se caracteriza por construirse desde y para la práctica, pretende mejorar la práctica desde su transformación al mismo tiempo que la comprende, demanda la participación de los sujetos en las mejoras de su propia comunidad, exige una actuación grupal en la que los sujetos participan en todas las fases de la investigación y se configura como una espiral de ciclos de planeación, acción, observación y evaluación. La I-A no pretende comprobar hipótesis o tomar datos para sacar conclusiones, sino que apunta a la transformación de una realidad cambiando tanto al investigador como a las situaciones en que este actúa.

Rojas (2012) menciona que no hay otra forma de hacer I-A diferente a la colaboración, en la que tanto los investigadores como los investigados tienen voz y participación en las diferentes etapas del estudio y de la misma manera son beneficiados con los cambios que se proponen a partir de las observaciones.

\section{Proceso de la investigación-acción}

Según la Escuela Nacional de Salud Pública de Cuba (Ensap, 2002), la I-A se produce en un bucle recursivo entre planeamiento, acción, observación y reflexión, esto implica que estos momentos no son lineales y totalmente estructurados, sino que se requiere de flexibilidad para replantear lo que sea necesario mientras se va y se viene en el bucle. Cada uno de los momentos se entiende conforme lo señala la Ensap (2002) de la siguiente manera:

- Planeación: es el momento que por definición precede a la acción y en donde esta se describe de manera organizada; sin embargo, la planeación debe tener en cuenta que toda acción social es de cierta manera impredecible y arriesgada, por lo que debe ser flexible y acomodarse una vez se regrese en el bucle 
- Acción: la acción está guiada por la planeación, una acción utiliza como plataforma la acción anterior, por lo que estas se encuentran relacionadas. A pesar de la planeación, la acción no está totalmente controlada, en lo esencial es arriesgada y toma lugar en tiempo real y con limitaciones materiales y políticas reales, muchas de ellas producto de los cambios sociales normales, por lo que la acción debe tener una cualidad tentativa y provisional, además de ser flexible para adaptarse a los cambios. En este punto es importante mencionar que la I-A difiere de la acción en que esta es observada y se recolecta información para ser analizada

- Observación: debe realizarse pensando en construir una base documental para su posterior reflexión, pero no debe ser demasiado rígida; al igual que los pasos anteriores debe ser lo suficientemente flexibles para no dejar afuera aquello que sucede inesperadamente, por ello se sugiere realizar los registros en un diario.

- Reflexión: además de rememorar la acción tal como quedó registrada en la observación, es un proceso activo que toma en cuenta las diferentes perspectivas que se presentaron, le halla sentido a los problemas, procesos y restricciones que se dieron en la acción, por lo general este momento es nutrido por las ideas de los participantes.

Una vez se ha realizado esta primera etapa, que como se mencionó no necesariamente es tan lineal como se describe, el bucle regresa, y la reflexión permite realizar nuevas planeaciones y ajustar las acciones.

\section{Tipología de investigación-acción}

Hay varios teóricos que han realizado propuestas referentes a los tipos de investigación acción teniendo en cuenta diferentes aspectos; sin embargo, la propuesta de Grundy (1982, 1991, citado en Baucela, 2004) es la que se expondrá a continuación: 
- Técnico: las acciones son orientadas por expertos y se ejecutan teniendo en cuenta esquemas preconcebidos, direccionándose hacia determinados resultados. Se orienta a mejorar las acciones y la eficacia del sistema.

- Práctico: se orienta a la indagación y reflexión de la práctica a la luz de sus fines; y viceversa, la reflexión de los fines y valores a la luz de la práctica. Se orienta a la comprensión de la realidad.

- Crítico: parte de la idea de que no siempre se puede realizar la modalidad práctica, por las limitaciones institucionales e ideológicas; por lo tanto, es necesario plantearse la transformación de estas limitaciones, no solamente pensar en la acción, la manera de sobrellevar esos obstáculos es recurriendo a las fuentes teóricas que sirvan de toma de conciencia a esas limitaciones de la práctica. Se orienta a participar en la transformación social.

Esta investigación se encuentra dentro del tipo crítico de I-A debido a que la intención de este trabajo es participar en un contexto realizando la indagación de una situación —arteterapia y su impacto en la vida de los estudiantes-, comprendiendo una parte de la realidad de la población con la que se trabajó para hacer un aporte a la transformación de esa realidad.

\section{La investigación-acción en educación superior}

Este método de investigación, que parte de la acción para el cambio y la reflexión de estos procesos, es frecuentemente utilizado en educación de diferentes niveles; ofrece la posibilidad de revisar las acciones emprendidas por docentes y estudiantes y ajustarlas en beneficio de la comunidad estudiantil y académica en general. En lo referente a la I-A en educación, Rincón (1997, citado en Baucela, 2004) menciona que es uno de los modelos de investigación más adecuados para fomentar la calidad y promover la reflexión y la educación continua en el profesional. 
Colmenares y Piñero (2008) hacen referencia a que la I-A se desarrolló en dos vertientes, una de ellas se orienta a hacia la sociología y la otra hacia la educación, en la cual aportaron investigadores como Freire, Stenhouse, Elliot, Carr y Kemmis. En Colombia, Bernardo Restrepo ha desarrollado diferentes I-A en el contexto educativo y ha realizado la distinción entre I-A educativa —orientada a la indagación y transformación en procesos escolares en general- y la I-A pedagógica —orientada a la formación de los docentes-.

Suárez Pazos (citado en Colmenares y Piñero, 2008) menciona que en el contexto educativo la I-A es una forma de indagar, explorar una situación con el ánimo de mejorarla, por lo que se presenta no solo como una herramienta de investigación, sino como una herramienta epistémica para el cambio educativo.

Colmenares y Piñero hacen mención sobre la importancia de la I-A en el contexto educativo:

La investigación-acción constituye una opción metodológica de mucha riqueza, ya que por una parte permite la expansión del conocimiento y por la otra va dando respuestas concretas a problemáticas que se van planteando los participantes de la investigación, que a su vez se convierten en co-investigadores que participan activamente en todo el proceso investigativo y en cada etapa o eslabón del ciclo que se origina producto de las reflexiones constantes que se propician en dicho proceso (2008).

Al pensar en el contexto educativo y en esta investigación, la I-A permite realizar planteamientos que flexibilicen el cómo de las asignaturas, el docente ajusta sus acciones de acuerdo con las respuestas del grupo de estudiantes, buscando así una mejora continua de los métodos de enseñanza y los procesos de aprendizaje. En el caso particular de la arteterapia, se va ajustando la sesión al ritmo y estado de los estudiantes y a los impactos observados en ellos.

Este proceso, según Schön (1983, citado en Colmenares y Piñero, 2008), implica pasar de un conocimiento práctico más bien inconsciente a un conocimiento reflexionado, crítico y teórico construido a 
través del dialogo entre colegas, docentes y estudiantes, lo que le da al conocimiento pedagógico una dimensión más social.

La I-A en educación es una invitación a construir conocimiento donde los diferentes actores se ven involucrados no solo como sujeto que investiga y sujeto investigado, sino que todos aportan ideas, visiones, buscan opciones y la manera de influir en su propio grupo, por lo que se tiene en cuenta una jerarquía más horizontal y menos vertical, siendo esta última la tradicional en investigación. Es de esta manera que los propios estudiantes se encargan de revisar y replantear acciones para su asignatura, en este caso la arteterapia, y las observaciones de los estudiantes en conjunto con las del docente/investigador, ayudan a replantear y elegir nuevos esquemas que faciliten los procesos internos y humanos del estudiante para su crecimiento y desarrollo personal. Teniendo en cuenta esta idea, es importante revisar algunas características puntuales de la I-A en el contexto educativo.

Rojas (2012) postula algunas características del mencionado método de investigación y, según menciona la autora, estas fueron propuestas por Elliot:

- Resuelve problemas de los profesores y lleva a la práctica valores educativos.

- Favorece procesos de reflexión sobre los medios y fines pedagógicos; supone que reflexionar sobre la calidad de la enseñanza implica pensar en los procesos y formas de aprendizaje más que en los resultados.

- Promueve prácticas reflexivas y desarrolla una actitud permanente de autoevaluación profesional.

- Integra la teoría y la práctica como elementos indisolubles en la vida del maestro

- Promueve que los docentes reflexionen sobre sus problemas y los resuelvan como colectivo profesional, por lo que al realizar cambios se incluye lo institucional, lo normativo y lo cultural. 
Por otra parte, Colmenares y Piñero (2008) mencionan algunas distinciones propias del contexto educativo cuando se pretende utilizar la I-A:

- Objeto de estudio: se exploran de manera natural los actos que suceden tanto dentro como fuera del aula; estos actos pueden ser de tipo pedagógico, administrativo, de gestión, de acción comunitaria, entre otros. Además de esta exploración hay que proponer acciones de cambio que experimentarán los actores del contexto educativo, por lo que no se tiene en cuenta solamente el problema en teoría o lo que se conoce de él por parte de los académicos y es importante para ellos.

- Intencionalidad: la finalidad, el objetivo de la I-A en educación, es mejorar la práctica, al mismo tiempo que se mejora la comprensión que de ella se tiene y de los contextos en que se realiza (Carr y Kemmis, 1988, citados en Colmenares y Piñero, 2008). Este es un trabajo que se realiza de manera conjunta, los diferentes actores reflexionan en torno al quehacer y el ser de la educación en diferentes niveles según los objetivos de cada investigación y así construyen significados compartidos y pueden modificar su realidad contextual. Es importante tener en cuenta que cualquier tipo de cambio afecta de diferentes maneras a los actores, por lo que estos cambios deben ser pensados teniendo en cuenta a quiénes beneficia y a quiénes puede perjudicar, todo ello en coherencia con los valores educativos y, por su puesto, estar atentos a los cambios colaterales no previstos.

- Los actores y los investigadores: en términos del contexto educativo, los investigadores no son solo los expertos que diseñaron la investigación, los investigados también son investigadores; docentes, estudiantes, directivos, administrativos, por lo que ellos también pueden tomar decisiones. Hay I-A que incluyen también a personas externas a la institución en que se está investigando, pero que están relacionados con ella, como padres de familia, personas de la comunidad en la que se encuentra 
la institución, organismos públicos —secretarías de educación, ministerios de educación-, entre otros.

- Los procedimientos: es importante aclarar que si bien es usual que los docentes reflexionen sobre sus prácticas educativas, sus sesiones de clase y con base en ello realicen modificaciones, esto no quiere decir que ellos estén siempre y sin saberlo, utilizando la I-A; para su utilización se requiere de un procedimiento sistemático, basado en la recolección de observaciones de manera organizada, que son producto de las vivencias de los diferentes participantes y que posteriormente serán analizadas con algún sistema definido. Para la recolección de la información pueden utilizarse diferentes técnicas, como los registros anecdóticos, diarios o notas de campo, fotografías, videos, relatos autobiográficos, grupos focales, observadores externos, descripciones ecológicas del comportamiento, entrevistas, cuestionarios, pruebas de rendimiento de los estudiantes - como los exámenes-, estudios de caso, entre otros. La elección de la técnica dependerá de las necesidades de investigación, y la información recogida debe ser sistematizada y analizada en categorías para poder tener resultados, para luego revisar si se están cumpliendo los objetivos; además de realizar las propuestas de cambio - acción - teniendo un conocimiento sólido, basado en la investigación de la situación.

Además de estas distinciones propias de la I-A en educación, hay algunos pasos sugeridos que también están aterrizados a este contexto. Hay varios autores que trabajan con respecto al tema y proponen diferentes momentos de la I-A en educación, sin embargo, a continuación se profundizará en la propuesta realizada por Elliot (citado en Rojas, 2012), quien dice que el proceso de I-A en lo educativo se divide en tres grandes fases:

- Identificación de una idea general: se parte de una primera recolección de información utilizando la técnica más conveniente, 
para realizar un diagnóstico que dé cuenta de la situación del grupo con el que se va a trabajar; es importante tener en cuenta las visiones y voces de los actores y realizar un consenso del área o tema en el que se va a intervenir.

- Exploración o planteamiento de las hipótesis de acción: estas hipótesis se entienden como aquellas ideas que se tienen para poder implementar y mejorar la situación del grupo, son las opciones de acción. Después del diagnóstico, el colectivo propone ideas que encierran sus valoraciones acerca de cómo y para qué mejorar la práctica de la enseñanza.

- Construcción de un plan de acción: este plan se genera a la luz del problema inicial en combinación con los diálogos que tienen los participantes durante el proceso investigativo. Debe ser un plan claro en cuanto a los medios y los recursos por utilizar para generar las acciones sobre el problema diagnosticado, generar las herramientas para recopilar información y contener sistemas de evaluación y seguimiento permanentes para cuidar la rigurosidad de la investigación y asegurar la participación de los actores.

Para cerrar este capítulo es importante mencionar que la I-A fuera de permitir la participación igualitaria del grupo educativo con el que se esté trabajando y darle voz en la realización y evaluación de cambios, trae consigo aportes a la experiencia del docente. Según Rojas (2012), la I-A es un método que profesionaliza la acción docente, que lo dota de una mirada analítica y reflexiva además de favorecer la modificación de las prácticas sociales y pedagógicas, por lo que es necesario que los docentes encuentren la manera de vincular la teoría con la práctica, de lo contrario sus acciones en el contexto educativo difícilmente mejorarán.

De esta manera, se espera con esta investigación realizar un aporte a la forma en la que se trabajan las asignaturas que están relacionadas con el desarrollo personal del estudiante y, de manera indirecta, del docente; partiendo de un método organizado que le permita a este 
último reflexionar sobre su que hacer y sobre la manera en que la asignatura de arteterapia se desarrolla y facilita procesos internos en el estudiante, para así modificarla recursivamente dirigiéndola hacia cambios benéficos para la comunidad universitaria y en mayor medida para la sociedad colombiana.

\section{Modelo educativo de la Universidad Minuto de Dios}

Esta investigación se realizó en un contexto de educación superior, por ello es importante establecer algunas características de la institución en la que se trabajó para comprender la manera como se articuló la cátedra de arteterapia dentro de los valores institucionales y el modelo pedagógico. Las descripciones que se presentan a continuación fueron tomadas de algunos documentos realizados por la Universidad Minuto de Dios (Uniminuto) en los que explican su modelo institucional.

El modelo educativo de la Uniminuto se concibe como un modelo praxeológico, lo que se entiende como el actuar humano reflexivo, es decir, se busca formar estudiantes que no actúen de manera impulsiva o basados solo en conocimientos teóricos, sino que puedan orientarse a cambiar su contexto buscando el beneficio personal y social. Este enfoque se centra en la educación integral, la cual apunta a desarrollar de manera armónica las diferentes dimensiones de la persona. Este modelo se enfoca por lo tanto a buscar que la persona se forme al combinar el saber — teoría- con la práctica - praxis-. La Uniminuto incorpora esta visión a todos sus programas mediante tres campos de formación:

1. Desarrollo humano: es inherente a la filosofía institucional y busca el equilibrio entre la formación académica y el proyecto de vida personal mediante un proceso cognitivo y de realización personal que apunta al desarrollo de todas las potencialidades de la persona permitiéndole ganar las competencias adecuadas para su opción personal y profesional. 
2. Responsabilidad social: se expresa mediante una responsabilidad consciente y crítica frente a las problemáticas de las comunidades y el país; este campo se construye mediante un trabajo praxeológico, investigativo y de realización personal, el cual se ve materializado en las prácticas sociales y profesionales e integra el conocimiento adquirido durante la carrera con la situación real de las comunidades, que requiere de responsabilidad y compromiso.

3. Competencias profesionales: se logra a través de un proceso cognitivo e investigativo que vincula la teoría y la práctica y pretende la transformación de las personas, sus conocimientos, habilidades y destrezas, generando la actitud permanente de "aprender a aprender" y el hábito reflexivo, crítico e investigativo en un contexto de interdisciplinariedad, además de aplicación del conocimiento para interpretar y transformar la sociedad.

Figura 4. Campos de formación Uniminuto

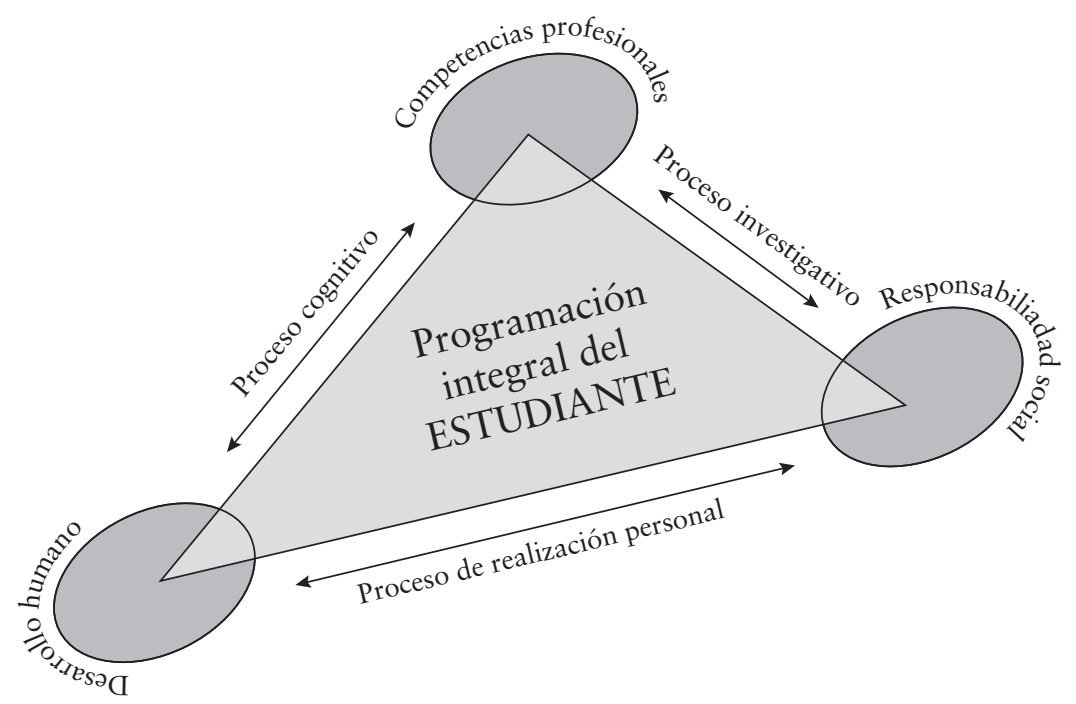

Fuente: tomado de www.uniminuto.edu 
Para la organización del modelo pedagógico, la estructura curricular se dividió en cuatro componentes curriculares, cada uno está organizado por áreas, núcleos o ejes, los cuales serán definidos según lo establezca la respectiva área del conocimiento; estas, a su vez, están compuestas por créditos obligatorios o electivos, previamente definidos por los comités curriculares de cada programa. A continuación se presenta la estructura curricular de la Uniminuto:

Figura 5. Modelo curricular Uniminuto

1. Componente básico profesional Porcentaje de créditos sobre el total de la carrera: $30 \%$ universitarios, $25 \%$ tecnologías Temas relacionados:

- General: competencias transversales para todos los programas de Uniminuto.

- Específico: competencias básicas iniciales comunes a todos los programas de la facultad o propias del programa

3. Componente profesional Porcentaje de créditos sobre el total de la carrera: $38 \%$ universitarios, $50 \%$ tecnologías Temas relacionados:

- Formación específica profesional

- Investigación formativa
2. Componente Minuto de Dios

Porcentaje de créditos sobre el total de la carrera:

$17 \%$ universitarios, $10 \%$ tecnologías Temas relacionados:

- Formación humana

- Formación social

- Formación en emprendimiento

4. Componente profesional complementario

Porcentaje de créditos sobre el total de la carrera: $15 \%$ universitarios, $15 \%$ tecnologías Temas relacionados:

- Práctica profesional

- Opción de grado

- Opciones complementarias (electivas)

Fuente: tomado de www.uniminuto.edu

Esta estructura curricular muestra los énfasis que se realizan en la formación de los estudiantes: el primero de ellos se orienta a la competencia básica profesional y tiene que ver con conocimientos afines a todos los programas que promueven habilidades básicas en el estudiante; el segundo hace referencia a la dimensión humana, social y de emprendimiento; el tercero es el componente profesional relacionado con la formación específica del programa en el que el estudiante se esté formando, y el cuarto es un componente profesional complementario que se presenta para cerrar el ciclo de formación del estudiante y se refiere a sus prácticas profesionales y su grado. 
Es importante mencionar que la investigación es donde la Corporación Universitaria Minuto de Dios vincula la teoría y la práctica, y tiene como campo principal de investigación el desarrollo humano y el desarrollo social sostenible; este se ramifica en cuatro subcampos en los que deben inscribirse todas las actividades de investigación que surjan de las facultades o de las unidades académicas, al igual que los proyectos de grado de los estudiantes. El objeto de las investigaciones no es la construcción de conocimiento generalizable, sino la autotransformación de quienes participan en concreto en cada situación educativa y de las comunidades a las que pertenecen o con las que investigan.

Se propende por una investigación de tipo praxeológico donde lo importante, para la Uniminuto a la hora de educar, es el proceso que viven los investigadores para llegar al conocimiento y no el conocimiento en sí, que enseñen para pensar, investigar y crear conocimiento de manera que se transforme la realidad. Es una investigación aplicada con enfoque interdisciplinario y centrada en la búsqueda del desarrollo humano y el desarrollo social.

Gracias al enfoque praxeológico se hizo posible la cátedra Taller Creativo, por medio de la cual se realizó esta investigación utilizando la arteterapia. Esta tiene como objetivo principal apoyar los procesos de autoconocimiento y autorregulación que le permitan al estudiante la toma de decisiones de manera reflexiva, lo que se articula muy bien con la praxeología, pues apunta a la formación del estudiante para que este actúe en el mundo teniendo en cuenta sus saberes y el contexto en que se encuentra, un actuar reflexionado.

\section{Fase de planeación}

El arte siempre ha sido y seguirá siendo una caja de sorpresas. Llegar a entender el potencial que hay detrás de la realización de cualquier obra de arte es comprender que no nos estamos enfrentando a una actividad cualquiera, sino que somos testigos de la creación y el reconocimiento de mundos que muchas veces ni siquiera los autores conocían hasta el momento de verlos plasmados en sus obras. 
Así como una obra se va formando poco a poco, este proyecto empieza con la iniciativa de la Facultad de Ciencias de la Comunicación, de la Universidad Minuto de Dios, de crear una Tecnología en Realización Audiovisual que cumpliera con los lineamientos de la universidad y que le permitiera a más jóvenes formarse y lograr sus sueños. Esta tecnología iba a tener un componente especial, una clase en la que, a través de medios alternativos, se pudieran disminuir los índices de deserción, violencia, comportamientos antisociales, propios de los estudiantes de esa Facultad, identificando alertas y remitiendo a los estudiantes a Bienestar Universitario cuando fuera necesario para darles las ayudas pertinentes, sobre todo al inicio de su proceso académico.

Es en este escenario donde surgió el Taller Creativo como un curso introductorio en formato de laboratorio-taller, el cual busca fomentar en los estudiantes el autoconocimiento con el fin de canalizar el material inconsciente en propuestas creativas. Este taller comenzó teniendo los siguientes tres módulos: sonido, movimiento y artes plásticas. Cada uno especialmente diseñado para lograr que el estudiante sea estimulado e inspirado a través de experiencias artísticas, creativas y terapéuticas, a trabajar directamente con su material interior - emociones, pulsiones, ideas inconscientes, ideas creativas-. El módulo de expresión plástica fue un escenario ideal para empezar a buscar estrategias artísticas que lograran la obtención de esos logros en el campo de la educación, y, enmarcadas en este escenario, se comenzaron a plantear preguntas de investigación referentes al impacto de la arteterapia en la vida de los jóvenes estudiantes.

Para empezar a plantear la propuesta tanto del taller como de la investigación, se hizo un primer intento de identificación de los estudiantes de la Facultad, pues era la primera cohorte de esta tecnología y no se tenían datos anteriores para generar un "plan de vuelo".

Se encontró que, en su mayoría, los estudiantes de la universidad son de estratos 2 y 3, y que quienes asisten a la Sede Principal y La Calle 90 son de Bogotá. En algunos casos los estudiantes pueden venir de poblaciones cercanas, aunque no son casos comunes debido a las sedes que la Uniminuto tiene en diferentes lugares del país. Hay estudiantes que trabajan en la mañana y estudian en la tarde o 
en la noche. Adicionalmente, algunos estudiantes se van en medios de transporte alternativos como patines, patineta, bicicleta, moto, entre otros, por el ahorro que representa frente al uso del transporte tradicional.

En el presente, después de cuatro años de haber abierto la Tecnología en Realización Audiovisual, el número de estudiantes aumenta cada vez más debido a diferentes factores, entre los cuales está la ideología de la universidad que expone como lema "Educación para Todos", buscando estrategias para la inclusión de todo tipo de personas sin filtros de admisión, como el resultado de las Pruebas Saber 11, entrevista o matrícula elevada. Esto exige del personal de la Uniminuto, no solo del Departamento de Bienestar, un constate seguimiento para lograr que todos los estudiantes que se matriculen en cualquier programa, finalicen su proceso académico con éxito. Otras estrategias utilizadas son el apoyo financiero y el acompañamiento psicológico.

Antes de empezar las clases y la investigación, se tuvieron reuniones con el director del programa, la decana de la Facultad y los tres profesores encargados de la asignatura para organizar un microcurrículo que pudiera responder a las necesidades de la población y de la Facultad. Después de unos meses de trabajo, se decidió que la organización, como se mencionó anteriormente, iba a ser por módulos y que cada módulo iba a constar de cinco sesiones. El orden de los módulos se dejó libre con el fin de empezar a probar cual sería el que más serviría al proceso. En otras palabras, las sesiones se organizaron de la siguiente manera:

- Sesión de inicio

- Cinco sesiones de artes plásticas

- Cinco sesiones de sonido

- Cinco sesiones de movimiento

- Sesión de cierre 
De manera particular, porque es la que corresponde a esta investigación, la sesión de artes plásticas integró a su plan de trabajo la arteterapia como estrategia pedagógica para permitir que los estudiantes tuvieran dos momentos: uno de catarsis, en donde dejan salir todo tipo de sentimientos y emociones sin ningún filtro ni juicio a través de la técnica que se les proponga; y otro momento en el que, después de sacar el material interior, pueden empezar un proceso de reconocimiento o autodescubrimiento. Vale la pena aclarar que no es un acompañamiento terapéutico individual, sino más bien una atención comunitaria pedagógica donde se brindan espacios y estrategias para que los mismos estudiantes sean los que se encarguen de validar sus emociones y su propio conocimiento.

Se entiende de esta manera que el objetivo de las sesiones es, más que buscar la sanación interior de los estudiantes, el que ellos mismos se expresen, se reconozcan e identifiquen la existencia de su propia realidad y la puedan nombrar. Si después de realizar este proceso pueden reconocer una liberación de ciertos conflictos internos, es ganancia para la experiencia tanto personal como de la investigación. Pero si por el contrario, se evidencia que el estudiante está sumido en un estado que no se puede manejar, es remitido inmediatamente al Departamento de Bienestar para que los psicólogos le den la ayuda oportuna.

Con este contexto se comienza a pensar en la necesidad de investigar la experiencia de la arteterapia en los jóvenes y cómo esta técnica puede ayudarles expresar y resolver cuestiones personales, por lo que es necesario plantear desde la metodología cualitativa una estrategia de investigación que permita evidenciar qué sucede con los jóvenes y la arteterapia. Es así como se define la I-A como método de investigación, donde el planteamiento de las fases permite un acercamiento al fenómeno de estudio y su reconfiguración con cada análisis de la experiencia. También se definen como instrumentos de recolección de información los trabajos de los estudiantes y los textos a los que ellos permitan acceder, toda vez que se consideran el discurso y los trabajos artísticos como posibilidad de expresar la vivencia de la arteterapia para el joven. 


\section{Fase de acción}

$\mathrm{Al}$ entrar en el grueso de las sesiones, es importante reafirmar que la metodología utilizada siempre proyectó la necesidad de una planeación que, en la mayoría de los casos, utiliza como material base la reflexión o evaluación del proceso anterior.

En este orden de ideas, las únicas clases que se planearon sin una experiencia previa fueron las realizadas en el 2011-1. Esto no quiere decir que no se haya tenido una base teórica y una experiencia pedagógica anterior para concertarlas. Solo que por ser las primeras generaron el proceso reflexivo de toda las demás y esto hace parte de la experiencia de la I-A.

Cada una de las cinco sesiones que se realizaron por semestre pasaron por un momento, previo a la acción, de descripción detallada de cada una de las actividades por realizar tanto para el desarrollo del taller como de la investigación. En un formato se establecían los tiempos, los espacios, los materiales, las técnicas por utilizar y unos posibles ejercicios por ejecutar en la clase, entendiendo, después del primer año de realización del taller, que toda acción, aún más si es social, es de cierta manera impredecible. Esto generó en la planeación, pero sobre todo en la práctica del taller, una flexibilidad sana y necesaria para acomodarse al grupo y sus propias necesidades. Las técnicas podían ser las mismas, pero la manera de abordarlas y motivarlas dependía del grupo que tomaba la sesión, por lo que era necesario acudir a la flexibilidad de la I-A.

El número de sesiones permaneció constante hasta el segundo semestre del 2013, cuando la clase fue cambiada para los lunes. Esto en otro país puede no tener relevancia, pero en Colombia, uno de los países con más festivos del mundo, si afectó el número de sesiones por semestre. Se redistribuyeron las sesiones obteniendo más o menos cuatro por módulo y definitivamente se tuvo que omitir la sesión de inicio.

Cada sesión, con una duración de tres horas, mantuvo la misma estructura: warming up o calentamiento, trabajo plástico, diálogo o espacio de socialización y cierre. 
El warming up o calentamiento (figura 6) se pensó para "aterrizar" a los jóvenes a la clase. Generalmente vienen de otras actividades académicas y llegan cargados y sin herramientas claras para empezar otra acción, entonces se dispone de quince minutos aproximadamente para que, por medio de diferentes actividades lúdicas, puedan moverse, estirarse, reírse, improvisar, pensar rápido, relajarse y pararse en el aquí y ahora de la sesión. Es un momento que disfrutan mucho porque los despierta y los llena de energía.

Figura 6. Actividad de calentamiento o warming up

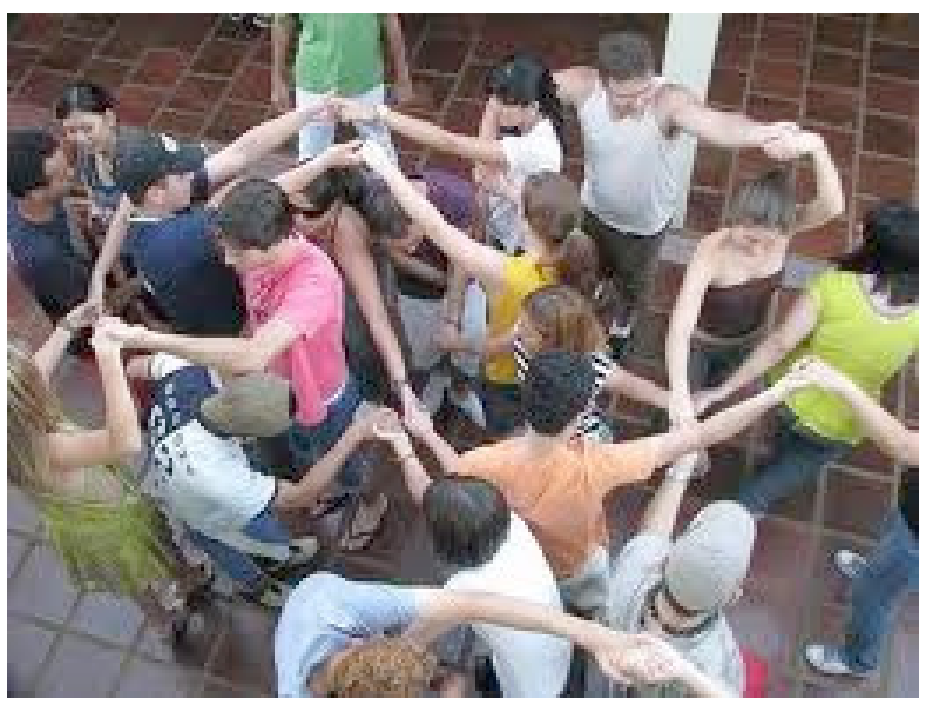

El trabajo plástico es el momento más extenso de la sesión y se podría decir que es uno de los más importantes. Antes de empezar el ejercicio plástico en sí, se realiza una práctica de concentración y receptiva que permite la plena presencia en los estudiantes. A través de la respiración se invita a estar plenamente conscientes para poder percibir lo que no es verbal y lo que está ocurriendo dentro de cada uno. Luego de un tiempo, en esta práctica se invita al estudiante a entrar en la consigna que se tiene preparada para esa sesión. En silencio y con los ojos cerrados se interioriza y se le permite al ser recordar, evocar, crear lo que quiera. 
Luego se plantea la actividad plástica por realizar y, si es necesario, se les brinda alguna teoría básica, bien sea para motivar la expresión o para direccionar la ejecución de la técnica.

Generalmente este tiempo se acompaña de un tipo de música que les permita concentrarse. Se pone para que todo el grupo pueda escucharla y se sugiere no tener dispositivos que interrumpan la clase. Luego de terminar el trabajo se destina un momento personal para intentar leer la imagen y escribir un texto casi espontáneo, tratando de generar un dialogo con la obra que resultó. Este es un momento muy especial, aunque los primeros semestres no fue muy nutrido y les resultaba difícil. Pero en el primer semestre del 2013 se hizo un cambio, la escritura creativa tomó el lugar del sonido, y esto ayudó a que los estudiantes tuvieran más herramientas para expresarse por este medio.

Figura 7. Trabajo plástico

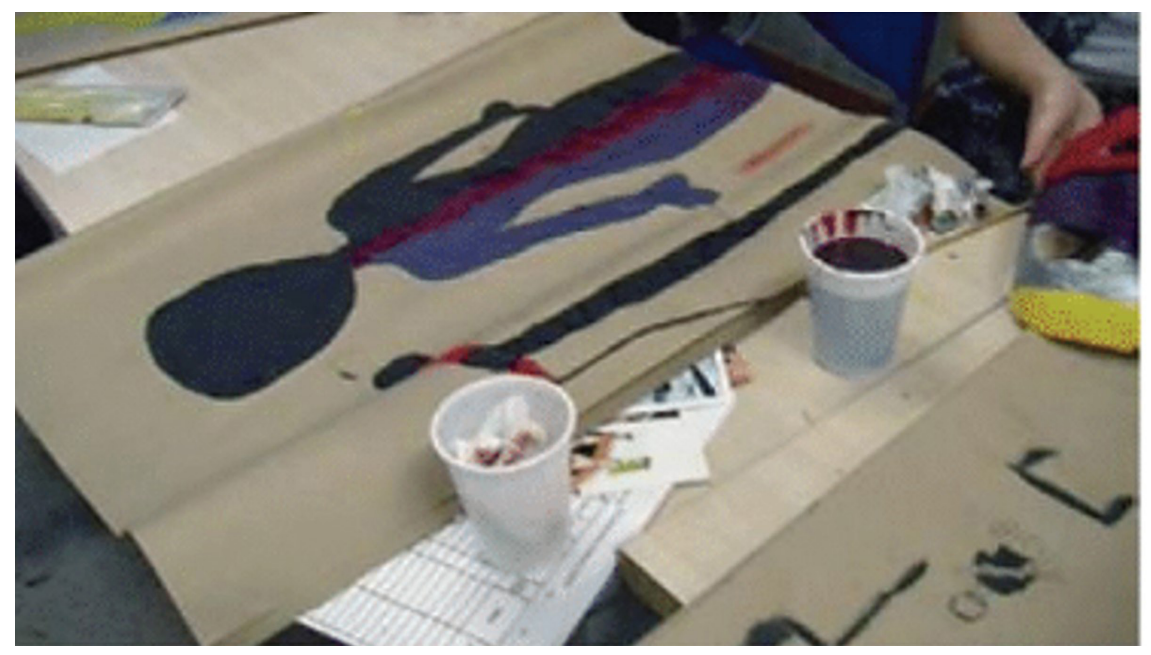

El momento del diálogo o socialización se realizaba los primeros semestres a través de una mesa redonda y la persona que quería compartía el texto escrito, la obra u otra experiencia. Pero con el paso del tiempo los grupos se fueron haciendo cada vez más grandes, pasando de diez a veinte personas. Esto hacía que las socializaciones fueran cada 
vez más extensas y difíciles. Entonces se tomó la decisión de empezar la socialización por parejas. Esta decisión disminuyó la ansiedad que algunos estudiantes experimentaban cuando se veían enfrentados al grupo completo y permitió además tener tiempos muy importantes de autoconocimiento y fortalecimiento de lazos interpersonales. Vale la pena añadir en este punto que se les daban a los estudiantes unas pautas básicas para la socialización, como el saber escuchar, el respeto, la curiosidad al momento de plantear preguntas para ayudar al descubrimiento del otro, no realizar juicios, no interpretar, estar pendiente del lenguaje no verbal, entre otras, que no solo les sirven para la socialización de las clases, sino para su propia vida.

En algunos casos se hizo necesario añadir otro espacio de diálogo entre actividades, nuevamente debido a las características del grupo.

Finalmente, el cierre es un momento relevante porque allí se entiende que el momento del proceso ya se dio y que paso a paso se va construyendo el camino del autoconocimiento.

El tiempo establecido para cada momento de la sesión se designaba de acuerdo con el grupo, con la actividad planteada y con la complejidad de la técnica.

Ahora, en cuanto a las técnicas empleadas, se tuvieron en cuenta las dimensiones de los materiales y los niveles de expresión e interacción con los medios de Lusebrink (2009). A partir de las teorías que ella plantea, se escogió trabajar primero los materiales más resistentes, como los lápices de colores y marcadores, con el fin de no generar ansiedad en los estudiantes al tener más control al momento de utilizarlos. Esa primera sesión se empieza siempre con un nivel de expresión totalmente cinestésico y sensorial para que tengan un primer acercamiento sin tanta presión frente al resultado.

En la mayoría de los semestres se trabajó el collage en la segunda sesión, pensando en la ventaja que les da a los estudiantes que no tienen facilidad para el dibujo y que aún tienen paradigmas frente al hecho de expresarse a través del arte, puesto que el collage es una técnica que brinda las imágenes hechas y solo hay que tomarlas. Pero desde el primer semestre del 2014 se decidió cambiarlo para el final de la sesión como un momento especial de cierre y conclusión del proceso que llevó cada estudiante, y como herramienta para mostrar los diferentes 
aspectos que siempre van a estar influyendo en la vida, aprovechando su carácter cognitivo.

Esta decisión se tomó pensando en el nivel de expresión de esta técnica y cómo se podría sacar más provecho de sus virtudes. Por eso, esta última experiencia funcionó muy bien y permitió sintetizar y recoger conclusiones sobre el proceso llevado en la clase, que salieron de los mismos estudiantes y enriquecieron la experiencia.

Para aportar al nivel sensorial se trabajó la arcilla, entendida como un material fluido, libre y con pocas instrucciones de uso. La poderosa arcilla tiene la virtud de ser estimulante, logrando hacer a la persona más consciente de lo interno al momento de trabajarla.

Aunque siempre el acercamiento al material se dio de manera absolutamente cinestésica, en los primeros semestres se trabajó de una manera muy controlada y racional. Esto no impidió que el material permitiera un diálogo y una representación, pero si situó la experiencia en un nivel que no era el apropiado, impidiendo sacar el mayor provecho de las sesiones.

A medida que fueron avanzando en los semestres, la arcilla fue tomando autonomía, pasando por un momento interesante donde se intentó articular el material con la técnica de animación cuadro a cuadro o stop motion. Esta transición permitió entender que la utilización de la arcilla podía llegar a ser más libre, pero ahora estaba el obstáculo de la animación. Esta técnica, aunque a los estudiantes les gustaba mucho, volcaba su interés a la calidad estética del trabajo, obviando el contenido interior que podía aflorar al momento de la experiencia. A eso hay que sumarle que los tiempos se volvían muy cortos y con ello no era posible dedicar el tiempo necesario a las socializaciones. En el 2014 la arcilla se mueve $100 \%$ en un nivel cinestésico-sensorial, evocando a través de las sensaciones las nociones más profundas. Es justo a ese punto donde se quería llegar con el material.

La cuarta técnica utilizada fueron las máscaras. Estas máscaras, entendidas como otra forma de modelar diferente a la de la arcilla, permitieron evidenciar el estado de las relaciones entre los miembros de los grupos. Además de ser una actividad relajada, se mueve en el 
nivel afectivo, logrando generar tanto en el que la hace como en la persona sobre la que la hacen, sensaciones y sentimientos que luego de las socializaciones son asociados a la manera como se interrelacionan y a su autoimagen.

Por último, con la pintura como técnica privilegiada para plasmar cualquier tipo de noción y que juega entre el nivel perceptual y el simbólico, el estudiante encuentra significados propios referenciales con ayuda del docente. Este material se trabaja primero de manera guiada para ayudar al estudiante que no tiene ningún acercamiento anterior o malas experiencias que no le permiten sentirse cómodo. Luego se prosigue con el trabajo libre, donde cada uno plasma lo que quiera sin juicios ni obstáculos.

Los cambios realizados respondieron a las necesidades de los estudiantes y de la investigación: después de los procesos de observación basados en la I-A se realizaban los cambios que eran necesarios para poder comprender mejor el proceso arteterapéutico en el joven y facilitar su experiencia de autoconocimiento y autorregulación.

La mayoría de los materiales utilizados en las clases los aporta el estudiante, es decir las cartulinas, las revistas, las tijeras, la arcilla y la gasa enyesada. Materiales como colores, marcadores, pegante, pintura y pinceles los aportó el docente para no cargar al estudiante con costos que no puede asumir. A partir del primer semestre del 2015 la totalidad de los materiales los aportó la Universidad.

Durante todas las sesiones se invita a los estudiantes a llevar un “diario creativo" donde se consigne la mayor información posible que resulte en el tiempo que duran las clases. Esto quiere decir que el diario no es privativo del taller sino que es un cuaderno donde cada estudiante es libre de expresar lo que sienta en el momento que quiera y de la forma que prefiera.

Hasta el 2013-1 las primeras sesiones y algunas posteriores, dependiendo del estudiante, se consignaron en el diario creativo debido a que, siendo la primera clase, todos los estudiantes juiciosamente llegaron con el diario. A partir del segundo semestre del 2013 se decide cambiar el carácter obligatorio del diario y proponerlo como una actividad provechosa exponiendo todos los beneficios que trae consigo 
el tener un registro de todo el proceso. Esto se hizo con el fin de desligar el hecho de llevar un diario, con la nota del semestre, porque se hacía evidente que los estudiantes pensaban en llenar el diario para mostrarle a profesor que sí estaban haciendo la tarea.

Sin embargo, durante todos los semestres se invita a los estudiantes a tener el diario y a llevarlo a la clase.

Al finalizar cada clase, y para obtener información para la investigación, además de la que registra la docente, se le solicitaba a los estudiantes que lo desearan un escrito referente a su experiencia en la sesión y que lo donaran a la investigación.

Después de describir un poco en qué consisten las sesiones es importante resaltar que durante el desarrollo de la clase la observación juega un papel primordial; no solo para poder responder efectivamente a las circunstancias particulares de la sesión, sino para construir una base que servirá para una posterior reflexión y planeación de las siguientes sesiones.

Cuando se analiza esa información para la construcción de la próxima intervención, los comentarios y textos de los estudiantes tienen un peso considerable, ya que hacen parte de las diferentes perspectivas que deben tenerse en cuenta al momento de evaluar los procesos.

Además de analizar los textos de los estudiantes, era necesario planear un protocolo de desarrollo curricular para la realización del Taller Creativo, evaluarlo semestre a semestre, modificarlo y así, al cabo de los cuatro años de investigación, realizar una propuesta que oriente la realización de procesos arteterapéuticos en contextos de educación superior. Por ello, es fundamental articular las voces de los estudiantes materializadas en los textos, la planeación y la reestructuración de las clases y el contexto de la educación superior.

Para esto se realizó un programa modelo por semestre y tres microcurrículos (ver anexo 1), los cuales tuvieron como objetivo servir de mapa orientador para el desarrollo de las clases y del Taller Creativo en el módulo de artes plásticas, y reflexionar sobre los cambios por realizar para los siguientes semestres, siguiendo la propuesta de la I-A.

A continuación se presentan los formatos de estas dos estructuras organizativas curriculares. 
Tabla 2. Estructura organizativa del Taller Creativo para cada semestre

\begin{tabular}{|c|c|}
\hline \multicolumn{2}{|c|}{ PROGRAMA MODELO - 1} \\
\hline Fecha de aplicación: & Nombre de la asignatura: \\
\hline N. ${ }^{o}$ total de horas: & N. ${ }^{\circ}$ de módulos por asignatura: \\
\hline \multicolumn{2}{|l|}{ Módulos: } \\
\hline N. ${ }^{\circ}$ de horas por módulo: & N. ${ }^{\circ}$ de sesiones por módulo: \\
\hline N. ${ }^{o}$ de horas por sesión: & Periodicidad de las sesiones: \\
\hline N. ${ }^{o}$ de estudiantes por semestre: & Salón: \\
\hline \multicolumn{2}{|l|}{ Profesores a cargo por módulo: } \\
\hline \multicolumn{2}{|l|}{ Horario: } \\
\hline \multicolumn{2}{|l|}{ Estructura de la sesión: } \\
\hline \multicolumn{2}{|l|}{ Momentos de evaluación: } \\
\hline \multicolumn{2}{|l|}{ Procedimiento de evaluación: } \\
\hline \multicolumn{2}{|l|}{ Técnicas plástico-visuales utilizadas: } \\
\hline \multicolumn{2}{|l|}{ Criterios de inclusión: } \\
\hline \multicolumn{2}{|l|}{ Propuestas complementarias: } \\
\hline Observaciones: & \\
\hline
\end{tabular}

Tabla 3. Estructura organizativa de las clases de cada sesión de artes plásticas

\begin{tabular}{|l|l|}
\hline \multicolumn{2}{|c|}{ DESARROLLO DE LAS SESIONES } \\
\hline Sesión $\mathbf{n} .^{{ }^{\circ}}$ & Nombre de la sesión: \\
\hline N. $^{\text {o }}$ total de horas: & Materiales: \\
\hline Actividades: \\
\hline Ajustes para tener en cuenta: \\
\hline
\end{tabular}

Estos dos modelos se realizan teniendo en cuenta las estructuras propuestas por la Universidad Minuto de Dios y respondiendo a las normas de calidad que rigen a la institución. La estructura del programa modelo se realiza antes de iniciar el semestre, ya que es el documento orientador de la materia, mientras que el formato de las sesiones, si bien estas tienen una planeación previa para realizar en cada clase, es alimentado después de realizar cada una; en ese momento es posible para la docente/investigadora narrar cuáles fueron las actividades 
realizadas, si se requirió algún cambio en el plan inicial de la sesión y se elabora una evaluación sobre el funcionamiento de la clase, el logro de objetivos, el impacto que tuvo en los jóvenes y de esta manera se analiza si es necesario realizar ajustes para el siguiente semestre en que se utilice esa técnica.

\section{Fase de interpretación de resultados}

Para el análisis de los resultados es importante aclarar que se consideraron como resultados del proceso arteterapéutico los trabajos de los estudiantes y los textos que ellos escribían después, tanto en su diario creativo como los relacionados con los trabajos que realizaban. Estos discursos dan cuenta del autoconocimiento del estudiante y de los procesos de autorregulación que ellos evidencian frente a la situación plasmada en el trabajo artístico y, por lo general, expresaban algún dilema que generaba inquietud en el joven.

Debido a lo íntimo de la narración de los estudiantes, y que para alguno de ellos era material muy privado, no se tuvo acceso al texto de ningún diario creativo, pero se contó para la investigación con los trabajos que algunos estudiantes quisieron entregar a la docente/investigadora y los textos que los acompañaban. De los cuatro años de trabajo con los estudiantes en el Taller Creativo, se seleccionaron de manera aleatoria 56 textos entre aquellos que los estudiantes quisieron compartir con la docente/investigadora, teniendo en cuenta que hubiera dos textos por cada técnica artística en cada semestre de trabajo. Sin embargo, no fue posible tener algún texto referente a la técnica de máscaras, pues los participantes no escribieron material extra con sus sensaciones, reflexiones o pensamientos al finalizar la tarea artística sino que lo hicieron en el diario creativo, situación que también se presentó para la técnica de dibujo durante los dos semestres del 2012 y el 2013-I.

Adicional a esta muestra, se enviaron vía correo electrónico las siguientes preguntas a los estudiantes que habían tenido la clase, para retomar así sus respuestas como textos de análisis: 
- ¿Cuál clase recuerda más?

- ¿Cuál fue su experiencia en esa clase?

- ¿Recuerda algo que generó un insight que haya repercutido hasta hoy?

De todos los estudiantes que habían participado en el taller y que recibieron el correo con las preguntas, solo respondieron tres. Debido a que fueron pocos los jóvenes que respondieron, se tomaron todas las respuestas y no se realizó muestreo; con estos últimos textos se estableció la muestra de textos para esta investigación.

Una vez fueron seleccionados se utilizó el análisis de contenido para comprender la información que habían proporcionado los estudiantes desde un nivel distinto. Esta técnica ha sido utilizada por ciencias como la Filosofía, la Sociología, la Antropología, Psicología y la Lingüística para comprender textos, sin embargo, la tarea de definirla resulta compleja, ya que dependiendo de los discursos por analizar, la ciencia que los analiza, el contexto y la investigación, el análisis del discurso varía. Es posible que la multiplicidad de formas de realizar la técnica mencionada esté relacionada con la variedad de concepciones de discurso, el cual según Íñiguez (2003) es un término polisémico, dependiendo de la ciencia, el autor, las inclinaciones de este, el contexto, entre otros aspectos, con que se entiende y se aborda el mismo.

Por las múltiples definiciones de análisis del discurso se retomarán tres que resultan útiles para comprender el ejercicio que se realizó. La primera de ellas es de Stubbs (1983, citado en Íñiguez, 2003), quien define la técnica como el intento de estudiar la organización del lenguaje por encima de la oración o la frase, por lo tanto, apunta al estudio de estructuras lingüísticas mayores, como la conversación o el texto; el análisis del discurso también se utiliza en contextos sociales y en el uso del lenguaje, más concretamente con la interacción entre personas.

Brown y Yule (1983, citado en Íñiguez, 2003) dicen que el análisis del discurso es el análisis de la lengua en su uso, por lo que no puede ser la descripción de formas lingüísticas con independencia de sus propósitos y funciones. 
Por último, Íñiguez y Antaki (1994, citado en Íniguez, 2003) definen el discurso como un conjunto de prácticas que promueven ciertas relaciones sociales y el análisis del discurso consiste en estudiar de qué manera esas prácticas actúan en el presente manteniendo y promoviendo esas relaciones.

Es claro entonces que en esta investigación no se realizará un estudio de estructuras lingüísticas o gramaticales, sino que se tendrán en cuenta los discursos de los jóvenes plasmados en forma de texto en un contexto, con sus significados, los cuales en este caso particular nos hablarán no solamente de las relaciones con otros según la manera en que se da el discurso, sino que también hablarán de las relaciones consigo mismos, de sus procesos de autoconocimiento y autorregulación.

Según Santander (2011), uno de los requisitos para realizar el análisis del discurso es definir el problema de investigación y delimitar el campo en el que se va a trabajar. En este caso se vincula al objetivo de esta investigación centrado en la arteterapia. Por otro lado, el autor agrega que es fundamental que la información por analizar sea de tipo discursivo, ya que pueden existir símbolos, signos, imágenes, representaciones, entre otros, que son susceptibles de ser analizados, pero se debe recurrir a otra técnica diferente.

En el análisis del discurso que aquí se realizará se utiliza un sistema de categorías, el cual, según Santander (2011), puede establecerse de dos formas según el interés del investigador: inductiva o deductivamente. En la primera, también llamada categorización emergente, se parte de no tener categorías previas al análisis del texto, por lo que el investigador se acerca a la información sin concepciones teóricas previas y basándose en los temas que ha encontrado en los textos define cuáles son las categorías; si opta por la categorización deductiva, partirá entonces de un marco teórico y unas hipótesis por revisar, por lo que definirá categorías que le ayuden a comprobar las hipótesis antes de analizar los textos.

En el caso de esta investigación, aunque se parte de un marco teórico que le da un contexto, la categorización fue inductiva; esta surgió de la revisión de los textos que escribieron los estudiantes y de los temas y los significados que más estaban presentes en ellos. 
Para el análisis de contenido de los textos se realizó una primera categorización haciendo uso del software Atlas Ti. Al ingresar los textos, se realizó un conteo de palabras identificando cuáles eran las que más se repetían en los escritos de los estudiantes, luego se agruparon por similitud, obteniendo un primer acercamiento a la categorización y una guía para revisar los textos hermenéuticamente, para lograr así la categorización definitiva.

Una vez se revisaron los textos con más detenimiento, se reagruparon ya no por palabras sino que se tuvieron en cuenta frases y su contexto, posteriormente se organizaron en la siguiente tabla:

Tabla 4. Organización de los textos seleccionados para el análisis de resultados

\begin{tabular}{|c|c|c|c|}
\hline Categoría & Descriptor & Texto & Observaciones \\
\hline $\begin{array}{l}\text { En este espacio } \\
\text { se ubica el } \\
\text { nombre de la } \\
\text { categoría a la } \\
\text { que pertenece } \\
\text { el texto. }\end{array}$ & $\begin{array}{l}\text { Es un tema } \\
\text { que aparece } \\
\text { de manera } \\
\text { significativa } \\
\text { dentro del } \\
\text { discurso de la } \\
\text { categoría y que } \\
\text { permite que esta } \\
\text { se evidencie. }\end{array}$ & $\begin{array}{l}\text { En este lugar } \\
\text { se plasman las } \\
\text { narraciones de } \\
\text { los jóvenes de } \\
\text { manera textual. }\end{array}$ & $\begin{array}{l}\text { En esta casilla se } \\
\text { realizan observaciones o } \\
\text { se aclara el contexto de } \\
\text { la frase de ser necesario } \\
\text { para comprender } \\
\text { por qué se ubicó en } \\
\text { determinada categoría. }\end{array}$ \\
\hline
\end{tabular}

Una vez se organizaron los textos por categorías fue posible comprender las diferentes alusiones que estos hacían, a qué áreas del autoconocimiento y de la autorregulación se inclinaban más los participantes y de qué manera impactó la arteterapia en su proceso en ese momento de su vida. Además, fue posible encontrar varias relaciones entre categorías y así darle mayor profundidad al proceso.

De manera transversal se realizaron reflexiones a lo largo de los cuatro años de investigación referentes al impacto del Taller Creativo y el direccionamiento para que permitieran procesos de autoconocimiento en los estudiantes, es por esto que desde la I-A se realizó no solo la investigación en arteterapia, sino que se pudo replantear la forma como se debían realizar las clases para lograr sintonizarse con el 
estudiante, sus necesidades y direccionarlos para descubrir, redescubrir y repensar aspectos de sus vidas; es por esto que se llevó a cabo el correspondiente análisis al programa modelo de cada semestre y al desarrollo de cada sesión. 